\title{
NETosis: A New Factor in Tumor Progression and Cancer-Associated Thrombosis
}

\author{
Melanie Demers, $\mathrm{PhD}^{1,2}$ Denisa D. Wagner, $\mathrm{PhD}^{1,2,3}$ \\ 1 Program in Cellular and Molecular Medicine, Boston Children's \\ Hospital, Boston, Massachusetts \\ 2 Department of Pediatrics, Harvard Medical School, \\ Boston, Massachusetts \\ ${ }^{3}$ Division of Hematology/Oncology, Boston Children's Hospital, \\ Boston, Massachusetts
}

Semin Thromb Hemost 2014;40:277-283.

\begin{abstract}
Address for correspondence Denisa D. Wagner, PhD, Program in Cellular and Molecular Medicine, Division of Hematology/Oncology, Boston Children's Hospital, Harvard Medical School, 3 Blackfan Circle, Third Floor, Boston, MA 02115 (e-mail: denisa.wagner@childrens.harvard.edu).
\end{abstract}

\begin{abstract}
Keywords

- NETs

- cancer

- thrombosis

- biomarkers

Neutrophils have long been known as innate immune cells that phagocytose and kill pathogens and mount inflammatory responses protecting the host from infection. In the past decades, new aspects of neutrophils have emerged unmasking their importance not only in inflammation but also in many pathological conditions including thrombosis and cancer. The 2004 discovery that neutrophils, upon strong activation, release decondensed chromatin to form neutrophil extracellular traps (NETs), has unveiled new avenues of research. Here, we review current knowledge regarding NETs in thrombosis, with a special focus on cancer-associated thrombosis as well as their potential role in cancer growth and metastasis. We discuss the prospective use of NET-specific biomarkers, such as citrullinated histone $\mathrm{H} 3$ and NET inhibitors, as tools to anticipate and fight cancer-associated thrombosis. We propose that the rapid developments in the field of NETosis may provide new targets to combat the thrombotic consequences of cancer and perhaps even help to contain the disease itself.
\end{abstract}

The scientific and medical community has in recent years increased its attention to neutrophils. New important functions and properties of these immune cells are emerging in addition to their role as the first line of defense against pathogens. The discoveries surrounding neutrophils promoted them from simple phagocytes to a sophisticated population of innate immune cells which orchestrate both the innate and adaptive inflammatory response., ${ }^{1,2}$ Among these novel characteristics, the formation of neutrophil extracellular DNA traps (NETs) has defined new roles for neutrophils in inflammation and immunity but also in pathological conditions including cancer biology and thrombosis.

\section{Neutrophil Extracellular Traps}

The first evidence that neutrophils release their chromatin in the extracellular space was provided in 2004 by Arturo Zychlinsky's group. ${ }^{3}$ The authors showed that upon activa- tion, neutrophils generate extracellular DNA fibers composed of granular proteases and nuclear constituents that trap and kill bacteria, thus defining a new form of antimicrobial innate response. Further in vitro characterizations described NETosis as a novel cell death program leading to the decondensation of the chromatin followed by nuclear and granular membrane disintegration, mixing of the components, and finally cytoplasmic membrane lysis and NETs release. ${ }^{4}$ In some cases, a more rapid ejection of the chromatin through vesicular exocytosis of nuclear contents was observed. ${ }^{5}$ In vivo, intact anuclear neutrophils that released only their nuclear contents without lysis were also observed. These "multitasking" cells retained the ability to crawl and phagocytose microbes in vivo. ${ }^{6}$ Recently, the term "vital NETosis" was proposed to distinguish it from NETosis by cell lysis ${ }^{4}$ and it has been suggested that different subsets of neutrophils or different stimuli could lead to one or the other. ${ }^{7}$
Issue Theme Cancer and Thrombosis: An Update; Guest Editor, Hau C. Kwaan, MD, FRCP.
Copyright $\odot 2014$ by Thieme Medical Publishers, Inc., 333 Seventh Avenue, New York, NY 10001, USA. Tel: +1(212) 584-4662.
DOI http://dx.doi.org/ 10.1055/s-0034-1370765. ISSN 0094-6176. 
The molecular mechanisms leading to NET formation remain unclear. The release of the chromatin by neutrophils was originally shown to be dependent on the generation of reactive oxygen species (ROS). ${ }^{4}$ The production of ROS occurs through the activation of nicotinamide adenine dinucleotide phosphate (NADPH) oxidase, RAF-MEK-ERK, and p38MAPK pathways ${ }^{8-10}$ whereas other stimuli activate NETosis in an NADPH oxidase independent manner. ${ }^{5,11}$ It has also been suggested that neutrophil elastase and myeloperoxidase are implicated in the process. ${ }^{12}$ Upon activation, neutrophil elastase translocates to the nucleus and helps chromatin decondensation by partially degrading histones. The chromatin decondensation is primarily driven by another modification of histones, histone citrullination through the activation of peptidylarginine deiminase 4 (PAD4). PAD4 (also called PADI4) is an enzyme highly expressed in many cancers $^{13}$ and in neutrophils where it is crucial to NET formation. ${ }^{14-16}$ The critical importance of PAD4 and histone citrullination has been documented by the use of PAD4 inhibition and treatment with a PAD4 fusion protein in HL-60 cells ${ }^{16}$ as well as by genetically engineered PAD4deficient mice whose neutrophils are unable to form NETs. ${ }^{14}$ Moreover, PAD4 overexpression in an osteosarcoma cell line was sufficient to induce chromatin decondensation and release, proving its function in this artificial setting. ${ }^{17}$ How PAD4 expression and activity are regulated in neutrophils and why NETosis sometimes results in only nuclear release and other times in cell lysis remain to be elucidated.

\section{Neutrophil Extracellular Traps in Thrombosis}

The ejection of neutrophil chromatin decorated with granular proteins and proteases at sites of infection traps and kills pathogens, conferring a protection to the host. However, the release of chromatin in the vasculature of an uninfected animal can be quite deleterious. Indeed, NETs promote thrombosis by providing a scaffold for platelet and red blood cell adhesion and aggregation ${ }^{18}$ and enhancing coagulation. ${ }^{19}$ Biomarkers of NETs have been observed in thrombi and plasma of baboons and mice subjected to deep vein thrombosis (DVT), revealing NETs as a structural part of the thrombus. ${ }^{18,20}$ The presence of NETs has also been seen in a human thrombus ${ }^{21}$ and in plasma of patients with DVT. ${ }^{22,23}$ It was suggested that thrombus neutrophils, through plateletdependent stimulation, were indispensable for the activation of factor XII and propagation of the thrombus in the inferior vena cava stenosis model of DVT in mice. ${ }^{24}$ Importantly, using PAD4-deficient mice, NETs were shown to be not only part of venous thrombi but to be critical to their formation and/or persistence. ${ }^{15}$ Thrombosis in PAD4-deficient mice can be rescued by wild-type neutrophil infusion showing their importance and sufficiency in the pathogenesis of DVT in mice. ${ }^{15}$ In humans, plasma markers of NETs correlate with thrombotic diseases activity such as thrombotic microangiopathies (TMA) ${ }^{25}$ and also coronary atherothrombosis. ${ }^{26}$

Exactly how NETs promote coagulation and thrombosis may depend on the circumstances in which they form. The major constituents of NETs, DNA, histones, and proteases, all have procoagulant properties. In vitro, extracellular nucleic acids, including genomic DNA, enhance protease activity of coagulation factors, ${ }^{27}$ and induce thrombin generation in platelet-poor plasma. ${ }^{28}$ In addition, histones are cytotoxic to the endothelium and can induce macro and microthrombosis in vivo. ${ }^{29}$ Histones inhibit anticoagulation of plasma by impairing thrombomodulin function, ${ }^{30}$ and promoting thrombin generation ${ }^{31}$ and platelet aggregation resulting in thrombocytopenia. ${ }^{32}$ Serine proteases, such as neutrophil elastase, have also been shown to inactivate tissue factor pathway inhibitor thus further increasing coagulation and fibrin deposition in vivo. ${ }^{19}$ Tissue factor has also been observed on NETs. ${ }^{33,34}$ Thus in many ways, the release of NETs in the vascular compartment triggers a procoagulant state and promotes binding and activation of platelets leading to thrombosis.

\section{Cancer and NETosis}

Neutrophils play a major role in cancer biology. ${ }^{35}$ They make up a significant portion of the inflammatory cell infiltrate in many mouse models of cancer and also in human tumors. ${ }^{36}$ However, their role in tumor progression is still debated because both pro- and antitumoral properties have been attributed to neutrophils. ${ }^{35,36}$ It was suggested that the opposing roles of tumor-associated neutrophils could be related to their stage of activation. ${ }^{36}$ Thus, NETs, which may be produced only by a subset of neutrophils, may potentially contribute to one of these properties. The release of the neutrophil's chromatin may influence many different steps of tumor development including tumor growth, angiogenesis, metastasis, and immune suppression. ${ }^{37}$ Our group reported the presence of a large area of dead neutrophils and NET-like structures in Lewis lung carcinoma (LLC) hemorrhagic tumors. ${ }^{38}$ A more detailed analysis of the LLC tumors by confocal microscopy clearly revealed the presence of large areas of neutrophil accumulation within the tumors (-Fig. 1A). These apparently necrotic areas are filled with not only intact neutrophils but also primed hypercitrullinated neutrophils ready to generate NETs and extracellular chromatin containing hypercitrullinated histones. A similar pattern was observed in histological sections of tumors of two patients with Ewing sarcoma. ${ }^{39}$ Thus, NETs are found in tumors at sites of neutrophil accumulation and may influence the cancer microenvironment. Whether neutrophils are recruited and NETs formed because of the inflammatory/ hypoxic environment of the tumor or NETs are responsible for the generation of necrotic areas remains to be clarified. Hypoxia within the tumor, for example, may activate hypoxia-inducible factor $1 \alpha$, which in turn may stimulate NETosis. ${ }^{40}$ Intriguingly, a recent study demonstrated that the phenotype of the tumor neutrophils is dependent on the tumor stage, becoming more tumor promoting as the tumor ages. ${ }^{41}$ The study shows that at an early stage of tumor development, the antitumoral neutrophils are localized around the tumor whereas at a later time point the protumoral neutrophils accumulate inside the tumor. As NETs are 

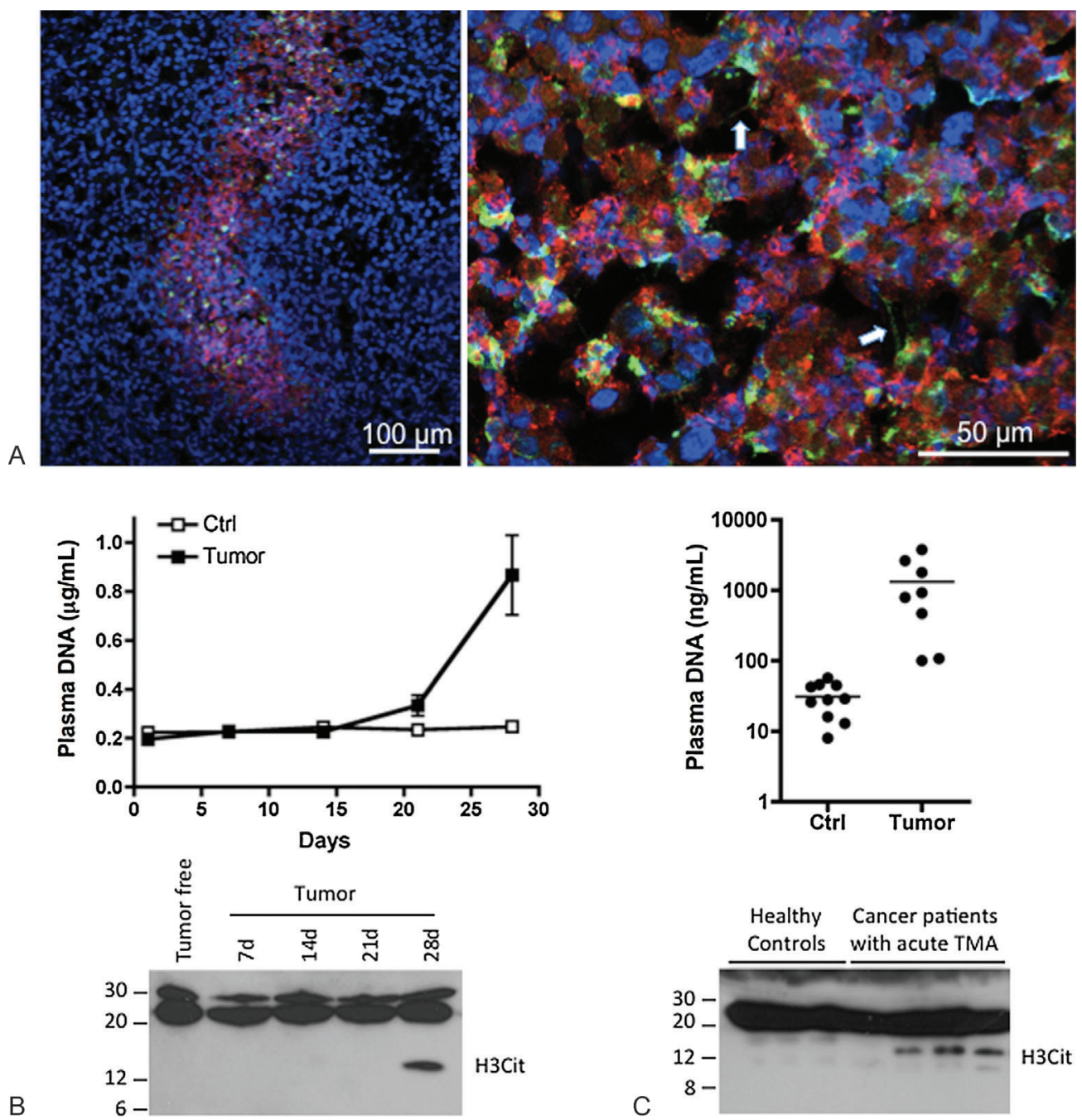

Fig. 1 Presence of neutrophil extracellular traps (NETs) in the tumor and in the plasma of tumor-bearing hosts. (A) Intratumoral presence of NETs. Lewis lung carcinoma tumor cells were injected subcutaneously in the right flank of C57BL/6 mice and tumors allowed to grow. At day 17 , mice were euthanized and tumors collected and snap-frozen. Forty micrometers sections were stained with anti-Ly6G (red, a neutrophil marker), anti$\mathrm{H} 3 \mathrm{Cit}$ (green) and DNA counterstained with Hoechst 33342 (blue). Confocal microscopy images were taken at least $5 \mu \mathrm{m}$ deep into the section to avoid artifacts. Low magnification representative image (left) shows a large area rich in neutrophils at the center of the tumor. H3Cit-positive neutrophils and extracellular chromatin are observed. High magnification image from this area (right) shows the presence of $\mathrm{H} 3 \mathrm{Cit}-\mathrm{positive}$ neutrophils but also extracellular H3Cit-containing strings of DNA (arrows). (B, C) High levels of plasma DNA and NET biomarkers are found in murine and human thrombotic hosts with cancer. (B) High levels of plasma DNA and H3Cit were found in mice with 4T1 breast cancer at a late stage of the disease. Reprinted with permission from Demers et al. ${ }^{58}$ (C). High level of plasma DNA and the presence of $\mathrm{H} 3 \mathrm{Cit}$ were also observed in most of the cancer patients with acute thrombocytic microangiopathies (TMA). The DNA results were adapted from Fuchs et al. ${ }^{25}$ This research was originally published in the journal Blood (Fuchs TA, Kremer Hovinga JA, Schatzberg D, Wagner DD, Lämmle B. Circulating DNA and myeloperoxidase indicate disease activity in patients with thrombotic microangiopathies. Blood 2012;120(6):1157-1164. @ American Society of Hematology). The method for $\mathrm{H} 3 \mathrm{Cit}$ analysis is described in Demers et al. ${ }^{58}$

observed within the tumor, this suggests an advantageous effect of NETs on primary tumor growth.

The involvement of NETs in promoting metastasis has recently been elegantly shown using intravital microscopy. ${ }^{42}$ In a model of systemic infection, a condition promoting metastasis, the authors documented NETs deposition on the microvasculature and subsequent trapping of circulating cancer cells in the DNA web. It was known that, during septicemia, NETs are released in the vasculature ${ }^{43}$ where they trap the bacteria. ${ }^{44}$ The authors hypothesized that, similar to the immobilization of bacteria, NETs would immobilize the tumor cells. Indeed, they show that NETs-entrapped tumor cells survive and proliferate to form nodules. ${ }^{42}$ It is possible that NETs or their degradation products are inhibiting immune cells allowing better survival. The observed increase in tumor metastasis again suggests a role for NETs in enhancing tumor progression. The effect of NETs could be attenuated by treatment with DNase, an enzyme that cleaves the DNA backbone of NETs. ${ }^{3}$ Thus, the presence of intravascular NETs following sepsis promotes metastasis in mice. Whether NETs just protect or anchor cancer cells physically or whether they generate thrombin that promotes tumor growth $^{45-49}$ remains to be determined. 


\section{Neutrophil Extracellular Traps in Cancer- Associated Thrombosis}

The association of cancer and thrombosis was reported in the 19th century by Armand Trousseau, describing thrombosis as a presenting feature even before the diagnosis of cancer; this is now defined as Trousseau syndrome. ${ }^{50}$ Over the years, studies have shown that tissue factor, microparticles, cytokines, soluble P-selectin, elevation in coagulation factors, secretion of mucins, thrombocytosis, and leukocytosis are implicated in the prothrombotic state associated with cancers. ${ }^{51-53}$ Interestingly, in 1986, Shoenfeld et al observed that the leukocytosis in 10 different types of nonhematologic malignancies was attributed mainly to an increase in polymorphonuclear cells and was associated with a poor clinical outcome. ${ }^{54}$ Indeed, recently, an increase in peripheral neutrophil counts and/or intratumoral neutrophils or a high neutrophil to lymphocyte ratio were all linked to poor prognosis and outcome in many different types of cancer. ${ }^{55}$ In humans, this elevation in neutrophils was seen mainly in renal cell carcinoma, melanoma, hepatocellular carcinoma, glioblastoma, colorectal, gastric, esophageal, lung, ovarian, and head and neck cancer, most of which are associated with a high risk of venous thromboembolism. ${ }^{56}$

Using a murine model of mammary carcinoma, in which a leukemoid reaction leading to an increase in blood neutrophil has been described, ${ }^{57}$ we recently showed occurrence of spontaneous thrombosis. ${ }^{58}$ Lung thrombosis was observed at a late stage of the disease when the neutrophil count and level of plasma DNA were extremely high (-Fig. 1B). Surprisingly, when neutrophils from tumor-bearing mouse blood were analyzed, an increasing number of highly hypercitrullinated neutrophils, ready to generate NETs, were observed as the tumor progressed. At the late time point where thrombosis was identified, a reduced number of highly hypercitrullinated neutrophils were counted and hypercitrullination of histone $\mathrm{H} 3$ (H3Cit) was detected in the plasma (-Fig. 1B), suggesting that NETosis was spontaneously occurring. ${ }^{58}$ The generation of NETs and the associated prothrombotic state can also be induced in tumor-bearing mice at an early time point of tumor progression by injection of low-dose lipopolysaccharides (LPS).

The predisposition of tumor-induced neutrophils to NET formation was confirmed in vitro in mammary and lung carcinoma as well as chronic myelogenous leukemia. ${ }^{58}$ The molecular mechanism leading to the priming of tumorinduced neutrophils seems to be related to an increase in plasma granulocyte colony-stimulating factor (G-CSF). Indeed, the elevation in neutrophil count, neutrophil priming, and the prothrombotic phenotype could be mimicked by administration of G-CSF before LPS in tumor-free mice. G-CSF-producing tumors have also been associated with poor prognosis. ${ }^{59-61}$

The thrombotic state produced upon NET generation seems to be related to the DNA scaffold. Digestion of NETs by pretreatment with DNase before LPS injection prevented thrombosis in tumor-bearing mice. ${ }^{58} \mathrm{High}$ levels of plasma DNA in cancer patients were described more than 30 years ago. ${ }^{62}$ Since then, an increase in plasma or serum DNA has been observed in many different types of cancer. ${ }^{63}$ The DNA was long thought to be released either from the tumor or the host-injured tissue by apoptotic or necrotic cell death. ${ }^{64}$ In fact, it has been shown that a large amount of nontumoral DNA is found in the plasma during tumor progression in rat models. ${ }^{65,66}$ High plasma DNA has also been observed in cases of inflammatory disease and trauma. ${ }^{63}$ Thus, the identification of NETs in cancer, as well as in other inflammatory diseases, now provides another explanation for the elevated plasma DNA. The association of plasma DNA with neutrophil counts and NETs biomarkers, such as H3Cit, could be used as diagnostic tools to evaluate propensity to thrombosis. Indeed, patients with tumorassociated TMA show high levels of plasma DNA ( - Fig. 1C) that are associated with NETs markers myeloperoxidase and calprotectin. ${ }^{25}$ Moreover, on further analysis, we now show that H3Cit can be detected in the stored plasma from most of these cancer patients with an acute episode of TMA whereas no H3Cit was found in the plasma of healthy controls ( - Fig. 1C). Thus, cell-free H3Cit can be observed in the plasma of cancer patients with thrombotic complications, just as it was observed in mice. ${ }^{58}$ This emphasizes H3Cit's potential as a biomarker for cancer-associated thrombosis. The contribution of NETs to the total level of plasma DNA found in cancer hosts remain to be addressed.

In our mouse models, tumor-induced neutrophils are sensitized to NET formation and can easily release their chromatin and promote thrombosis upon encountering a second hit even at an early stage of the disease. Thus, what happens after chemotherapy? It is known that chemotherapies induce cell death and release of DNA in the plasma and are associated with a high risk of thrombosis. ${ }^{67}$ Large DNA fragments are found in the plasma of patients after the first cycle of docetaxel chemotherapy for prostate cancer ${ }^{68}$ and neoadjuvant chemotherapy for breast cancer. ${ }^{69}$ The appearance of these large DNA fragments was attributed to rapid tumor cell necrosis following treatment. With our new knowledge on NETs, it is plausible that chemotherapies could either directly induce NETosis or serve as the second hit in NET generation. Indeed, Swystun et al reported that doxorubicin, epirubicin, and 5-fluororuacil, three breast cancer therapeutic agents, induce the release of DNA and thrombin-antithrombin complexes (TAT) when injected in healthy mice. ${ }^{28}$ However, in vitro, only doxorubicin and epirubicin had the potential to induce DNA release from isolated neutrophils suggesting that some drugs are indeed sufficient for NET induction. In vitro, in venous whole blood, the release of DNA could be prevented by the addition of the antioxidant glutathione, which prevents damage by ROS and thus NETosis. Moreover, the authors showed that the cell-free DNA released induces thrombin generation in whole blood, and that an increase in cell-free DNA and TAT are found in the plasma of early-stage breast cancer patients 24 hours postchemotherapy, ${ }^{28}$ a time point where thrombotic events are likely to occur. ${ }^{70}$ Thus, this study defined NETs as a novel procoagulant linking chemotherapy to thrombosis. ${ }^{70}$ 


\section{Conclusion}

The discovery of the formation of NETs by stimulated neutrophils unveiled unexpected functions of neutrophils in inflammatory diseases, cancer, and thrombosis. Many aspects of this new field still need to be addressed. Through the release of chromatin in tissues or in the vasculature, NETosis is responsible for increased inflammation/coagulation. It is also possible that, in turn, inflammation/coagulation activates neutrophils and induces them to form more NETs either directly or through the consequences of platelet activation. Would inhibition of coagulation prevent NETosis? Inversely, could the inhibition of NETs reduce the procoagulant state? Are NETs directly implicated in metastasis or is the general inflammation and thrombin generation triggered by NETs the main player?

The discovery of the presence of NETs within the tumor environment also suggests new leads for the development of diagnostic tools for NET detection and NET inhibition. For example, PAD4 inhibitors could be used in cancer patients to prevent tumor spread and cancer associated-thrombosis. Interestingly, the use of PAD inhibitor has already been assessed in settings of cancer. PAD4 is overexpressed in various human cancers ${ }^{71,72}$ and it interacts with $\mathrm{p} 53$ to repress the transcription of tumor suppressor genes. ${ }^{73}$ Therefore, the use of a pan PAD inhibitor $^{74,75}$ inhibits cancer cell proliferation ${ }^{73}$ and reduces tumor growth. ${ }^{76}$ Recently, Wang et al have designed a more potent inhibitor of PAD4, which also inhibits PAD2 to a lesser extent, and observed an even greater inhibition of tumor growth. ${ }^{77}$ Thus, in addition to their direct effect on cancer cells, the use of PAD4 inhibitors would also prevent PAD4-driven NETosis, thereby inhibiting not only tumor growth but also cancer-associated thrombosis. Of note, as the overexpression of PAD4 in a cancer cell line leads to the generation of NET-like structures, $^{17}$ and PAD4 is markedly overexpressed in many human cancers, the potential release of cancer cell chromatin, in addition to the neutrophils' extracellular chromatin, should be considered as it could also affect tumor progression and cancerassociated thrombosis. The presence of NETs within the tumor microenvironment and in the blood of tumor-bearing hosts has opened new avenues of research in cancer biology as well as provided new explanations for the interplay of cancer with inflammation and thrombosis.

\section{Acknowledgments}

This work was supported by National Heart, Lung, and Blood Institute of the National Institutes of Health grant R01 HL102101 (to D.D.W.). We thank Lesley Cowan and Kimberly Martinod for critical review of the article. We thank Bernhard Lammle and Johanna A. Kremer Hovinga for kindly providing the human cancer/TMA plasma samples and Kimberly Martinod for the western blot analysis. We also thank Siu Ling Wong for help with confocal microscopy.

\section{References}

1 Amulic B, Cazalet C, Hayes GL, Metzler KD, Zychlinsky A. Neutrophil function: from mechanisms to disease. Annu Rev Immunol 2012;30:459-489
2 Mócsai A. Diverse novel functions of neutrophils in immunity, inflammation, and beyond. J Exp Med 2013;210(7):1283-1299

3 Brinkmann V, Reichard U, Goosmann C, et al. Neutrophil extracellular traps kill bacteria. Science 2004;303(5663):1532-1535

4 Fuchs TA, Abed U, Goosmann C, et al. Novel cell death program leads to neutrophil extracellular traps. J Cell Biol 2007;176(2): 231-241

5 Pilsczek FH, Salina D, Poon KK, et al. A novel mechanism of rapid nuclear neutrophil extracellular trap formation in response to Staphylococcus aureus. J Immunol 2010;185(12):7413-7425

6 Yipp BG, Petri B, Salina D, et al. Infection-induced NETosis is a dynamic process involving neutrophil multitasking in vivo. Nat Med 2012;18(9):1386-1393

7 Yipp BG, Kubes P. NETosis: how vital is it? Blood 2013;122(16): 2784-2794

8 Bianchi M, Hakkim A, Brinkmann V, et al. Restoration of NET formation by gene therapy in CGD controls aspergillosis. Blood 2009;114(13):2619-2622

9 Hakkim A, Fuchs TA, Martinez NE, et al. Activation of the Raf-MEKERK pathway is required for neutrophil extracellular trap formation. Nat Chem Biol 2011;7(2):75-77

10 Keshari RS, Verma A, Barthwal MK, Dikshit M. Reactive oxygen species-induced activation of ERK and p38 MAPK mediates PMAinduced NETs release from human neutrophils. J Cell Biochem 2013;114(3):532-540

11 Parker H, Dragunow M, Hampton MB, Kettle AJ, Winterbourn CC. Requirements for NADPH oxidase and myeloperoxidase in neutrophil extracellular trap formation differ depending on the stimulus. J Leukoc Biol 2012;92(4):841-849

12 Papayannopoulos V, Metzler KD, Hakkim A, Zychlinsky A. Neutrophil elastase and myeloperoxidase regulate the formation of neutrophil extracellular traps. J Cell Biol 2010;191(3):677-691

13 Wang S, Wang Y. Peptidylarginine deiminases in citrullination, gene regulation, health and pathogenesis. Biochim Biophys Acta 2013;1829(10):1126-1135

14 Li P, Li M, Lindberg MR, Kennett MJ, Xiong N, Wang Y. PAD4 is essential for antibacterial innate immunity mediated by neutrophil extracellular traps. J Exp Med 2010;207(9):1853-1862

15 Martinod K, Demers M, Fuchs TA, et al. Neutrophil histone modification by peptidylarginine deiminase 4 is critical for deep vein thrombosis in mice. Proc Natl Acad Sci U S A 2013;110(21): 8674-8679

16 Wang Y, Li M, Stadler S, et al. Histone hypercitrullination mediates chromatin decondensation and neutrophil extracellular trap formation. J Cell Biol 2009;184(2):205-213

17 Leshner M, Wang S, Lewis C, et al. PAD4 mediated histone hypercitrullination induces heterochromatin decondensation and chromatin unfolding to form neutrophil extracellular trap-like structures. Front Immunol 2012;3:307

18 Fuchs TA, Brill A, Duerschmied D, et al. Extracellular DNA traps promote thrombosis. Proc Natl Acad Sci U S A 2010;107(36): 15880-15885

19 Massberg S, Grahl L, von Bruehl ML, et al. Reciprocal coupling of coagulation and innate immunity via neutrophil serine proteases. Nat Med 2010;16(8):887-896

20 Brill A, Fuchs TA, Savchenko AS, et al. Neutrophil extracellular traps promote deep vein thrombosis in mice. J Thromb Haemost 2012;10(1):136-144

21 Nakazawa D, Tomaru U, Yamamoto C, Jodo S, Ishizu A. Abundant neutrophil extracellular traps in thrombus of patient with microscopic polyangiitis. Front Immunol 2012;3:333

22 Diaz JA, Fuchs TA, Jackson TO, et al. Plasma DNA is elevated in patients with deep vein thrombosis. J Vasc Surg Venous Lymphat Disord 2013;1(4):341-348

23 van Montfoort ML, Stephan F, Lauw MN, et al. Circulating nucleosomes and neutrophil activation as risk factors for deep vein thrombosis. Arterioscler Thromb Vasc Biol 2013; 33(1):147-151 
24 von Brühl ML, Stark K, Steinhart A, et al. Monocytes, neutrophils, and platelets cooperate to initiate and propagate venous thrombosis in mice in vivo. J Exp Med 2012;209(4):819-835

25 Fuchs TA, Kremer Hovinga JA, Schatzberg D, Wagner DD, Lämmle B. Circulating DNA and myeloperoxidase indicate disease activity in patients with thrombotic microangiopathies. Blood 2012; 120(6):1157-1164

26 Borissoff JI, Joosen IA, Versteylen MO, et al. Elevated levels of circulating DNA and chromatin are independently associated with severe coronary atherosclerosis and a prothrombotic state. Arterioscler Thromb Vasc Biol 2013;33(8):2032-2040

27 Kannemeier C, Shibamiya A, Nakazawa F, et al. Extracellular RNA constitutes a natural procoagulant cofactor in blood coagulation. Proc Natl Acad Sci U S A 2007;104(15):6388-6393

28 Swystun LL, Mukherjee S, Liaw PC. Breast cancer chemotherapy induces the release of cell-free DNA, a novel procoagulant stimulus. J Thromb Haemost 2011;9(11):2313-2321

$29 \mathrm{Xu}$ J, Zhang X, Pelayo R, et al. Extracellular histones are major mediators of death in sepsis. Nat Med 2009;15(11):1318-1321

30 Ammollo CT, Semeraro F, Xu J, Esmon NL, Esmon CT. Extracellular histones increase plasma thrombin generation by impairing thrombomodulin-dependent protein $\mathrm{C}$ activation. J Thromb Haemost 2011;9(9):1795-1803

31 Semeraro F, Ammollo CT, Morrissey JH, et al. Extracellular histones promote thrombin generation through platelet-dependent mechanisms: involvement of platelet TLR2 and TLR4. Blood 2011; 118(7):1952-1961

32 Fuchs TA, Bhandari AA, Wagner DD. Histones induce rapid and profound thrombocytopenia in mice. Blood 2011;118(13): 3708-3714

33 Kambas K, Chrysanthopoulou A, Vassilopoulos D, et al. Tissue factor expression in neutrophil extracellular traps and neutrophil derived microparticles in antineutrophil cytoplasmic antibody associated vasculitis may promote thromboinflammation and the thrombophilic state associated with the disease. Ann Rheum Dis 2013. doi:10.1136/annrheumdis-2013-203430 [e-pub ahead of print]

34 Kambas K, Mitroulis I, Apostolidou E, et al. Autophagy mediates the delivery of thrombogenic tissue factor to neutrophil extracellular traps in human sepsis. PLoS ONE 2012;7(9):e45427

35 Brandau S, Dumitru CA, Lang S. Protumor and antitumor functions of neutrophil granulocytes. Semin Immunopathol 2013;35(2): 163-176

36 Gregory AD, Houghton AM. Tumor-associated neutrophils: new targets for cancer therapy. Cancer Res 2011;71(7):2411-2416

37 Demers M, Wagner DD. Neutrophil extracellular traps: A new link to cancer-associated thrombosis and potential implications for tumor progression. OncoImmunology 2013;2(2):e22946

38 Ho-Tin-Noé B, Carbo C, Demers M, Cifuni SM, Goerge T, Wagner DD. Innate immune cells induce hemorrhage in tumors during thrombocytopenia. Am J Pathol 2009;175(4):1699-1708

39 Berger-Achituv S, Brinkmann V, Abed UA, et al. A proposed role for neutrophil extracellular traps in cancer immunoediting. Front Immunol 2013;4:48

40 McInturff AM, Cody MJ, Elliott EA, et al. Mammalian target of rapamycin regulates neutrophil extracellular trap formation via induction of hypoxia-inducible factor $1 \alpha$. Blood 2012;120(15): 3118-3125

41 Mishalian I, Bayuh R, Levy L, Zolotarov L, Michaeli J, Fridlender ZG. Tumor-associated neutrophils (TAN) develop pro-tumorigenic properties during tumor progression. Cancer Immunol Immunother 2013;62(11):1745-1756

42 Cools-Lartigue J, Spicer J, McDonald B, et al. Neutrophil extracellular traps sequester circulating tumor cells and promote metastasis. J Clin Invest 2013 doi: 10.1172/JCI67484

43 Clark SR, Ma AC, Tavener SA, et al. Platelet TLR4 activates neutrophil extracellular traps to ensnare bacteria in septic blood. Nat Med 2007;13(4):463-469
44 McDonald B, Urrutia R, Yipp BG, Jenne CN, Kubes P. Intravascular neutrophil extracellular traps capture bacteria from the bloodstream during sepsis. Cell Host Microbe 2012;12(3):324-333

45 Green D, Karpatkin S. Role of thrombin as a tumor growth factor. Cell Cycle 2010;9(4):656-661

46 Hu L, Ibrahim S, Liu C, Skaar J, Pagano M, Karpatkin S. Thrombin induces tumor cell cycle activation and spontaneous growth by down-regulation of p27Kip1, in association with the up-regulation of Skp2 and MiR-222. Cancer Res 2009;69(8):3374-3381

47 Hu L, Lee M, Campbell W, Perez-Soler R, Karpatkin S. Role of endogenous thrombin in tumor implantation, seeding, and spontaneous metastasis. Blood 2004;104(9):2746-2751

$48 \mathrm{Hu}$ L, Roth JM, Brooks P, Ibrahim S, Karpatkin S. Twist is required for thrombin-induced tumor angiogenesis and growth. Cancer Res 2008;68(11):4296-4302

$49 \mathrm{Hu}$ L, Roth JM, Brooks P, Luty J, Karpatkin S. Thrombin up-regulates cathepsin D which enhances angiogenesis, growth, and metastasis. Cancer Res 2008;68(12):4666-4673

50 Varki A. Trousseau's syndrome: multiple definitions and multiple mechanisms. Blood 2007;110(6):1723-1729

51 Connolly GC, Khorana AA. Emerging risk stratification approaches to cancer-associated thrombosis: risk factors, biomarkers and a risk score. Thromb Res 2010;125(Suppl 2):S1-S7

52 Connolly GC, Khorana AA, Kuderer NM, Culakova E, Francis CW, Lyman GH. Leukocytosis, thrombosis and early mortality in cancer patients initiating chemotherapy. Thromb Res 2010;126(2): 113-118

53 Shao B, Wahrenbrock MG, Yao L, et al. Carcinoma mucins trigger reciprocal activation of platelets and neutrophils in a murine model of Trousseau syndrome. Blood 2011;118(15):4015-4023

54 Shoenfeld Y, Tal A, Berliner S, Pinkhas J. Leukocytosis in non hematological malignancies-a possible tumor-associated marker. J Cancer Res Clin Oncol 1986;111(1):54-58

55 Donskov F. Immunomonitoring and prognostic relevance of neutrophils in clinical trials. Semin Cancer Biol 2013;23(3):200-207

56 Paneesha S, McManus A, Arya R, et al; VERITY Investigators. Frequency, demographics and risk (according to tumour type or site) of cancer-associated thrombosis among patients seen at outpatient DVT clinics. Thromb Haemost 2010;103(2):338-343

57 DuPre' SA, Hunter KW Jr. Murine mammary carcinoma 4T1 induces a leukemoid reaction with splenomegaly: association with tumor-derived growth factors. Exp Mol Pathol 2007;82(1): $12-24$

58 Demers M, Krause DS, Schatzberg D, et al. Cancers predispose neutrophils to release extracellular DNA traps that contribute to cancer-associated thrombosis. Proc Natl Acad Sci U S A 2012; 109(32):13076-13081

59 Joshita S, Nakazawa K, Sugiyama Y, et al. Granulocyte-colony stimulating factor-producing pancreatic adenosquamous carcinoma showing aggressive clinical course. Intern Med 2009;48(9):687-691

60 Kaira K, Ishizuka T, Tanaka $\mathrm{H}$, et al. Lung cancer producing granulocyte colony-stimulating factor and rapid spreading to peritoneal cavity. J Thorac Oncol 2008;3(9):1054-1055

61 Kawaguchi M, Asada Y, Terada T, et al. Aggressive recurrence of gastric cancer as a granulocyte-colony-stimulating factor-producing tumor. Int J Clin Oncol 2010;15(2):191-195

62 Leon SA, Shapiro B, Sklaroff DM, Yaros MJ. Free DNA in the serum of cancer patients and the effect of therapy. Cancer Res 1977;37(3): 646-650

63 Fleischhacker M, Schmidt B. Circulating nucleic acids (CNAs) and cancer-a survey. Biochim Biophys Acta 2007;1775(1):181-232

64 Jahr S, Hentze H, Englisch S, et al. DNA fragments in the blood plasma of cancer patients: quantitations and evidence for their origin from apoptotic and necrotic cells. Cancer Res 2001;61(4): 1659-1665

65 García-Olmo DC, Picazo MG, Toboso I, Asensio AI, García-Olmo D. Quantitation of cell-free DNA and RNA in plasma during tumor progression in rats. Mol Cancer 2013;12:8 
66 García-Olmo DC, Samos J, Picazo MG, Asensio AI, Toboso I, GarcíaOlmo D. Release of cell-free DNA into the bloodstream leads to high levels of non-tumor plasma DNA during tumor progression in rats. Cancer Lett 2008;272(1):133-140

67 McMahon BJ, Kwaan HC. Thrombotic and bleeding complications associated with chemotherapy. Semin Thromb Hemost 2012; 38(8):808-817

68 Kwee S, Song MA, Cheng I, Loo L, Tiirikainen M. Measurement of circulating cell-free DNA in relation to $18 \mathrm{~F}$-fluorocholine PET/CT imaging in chemotherapy-treated advanced prostate cancer. Clin Transl Sci 2012;5(1):65-70

69 Deligezer U, Eralp Y, Akisik EE, et al. Size distribution of circulating cell-free DNA in sera of breast cancer patients in the course of adjuvant chemotherapy. Clin Chem Lab Med 2008; 46(3):311-317

70 Van Den Berg YW, Reitsma PH. Not exclusively tissue factor: neutrophil extracellular traps provide another link between chemotherapy and thrombosis. J Thromb Haemost 2011;9(11):2311-2312

71 Chang X, Han J. Expression of peptidylarginine deiminase type 4 (PAD4) in various tumors. Mol Carcinog 2006;45(3):183-196
72 Chang X, Han J, Pang L, Zhao Y, Yang Y, Shen Z. Increased PADI4 expression in blood and tissues of patients with malignant tumors. BMC Cancer 2009;9:40

73 Li P, Yao H, Zhang Z, et al. Regulation of p53 target gene expression by peptidylarginine deiminase 4 . Mol Cell Biol 2008;28(15): $4745-4758$

74 Luo Y, Knuckley B, Lee YH, Stallcup MR, Thompson PR. A fluoroacetamidine-based inactivator of protein arginine deiminase 4: design, synthesis, and in vitro and in vivo evaluation. J Am Chem Soc 2006;128(4):1092-1093

75 Jones JE, Slack JL, Fang P, et al. Synthesis and screening of a haloacetamidine containing library to identify PAD4 selective inhibitors. ACS Chem Biol 2012;7(1):160-165

$76 \mathrm{Li}$ P, Wang D, Yao H, et al. Coordination of PAD4 and HDAC2 in the regulation of p53-target gene expression. Oncogene 2010;29(21): 3153-3162

77 Wang Y, Li P, Wang S, et al. Anticancer peptidylarginine deiminase (PAD) inhibitors regulate the autophagy flux and the mammalian target of rapamycin complex 1 activity. J Biol Chem 2012;287(31): 25941-25953 